\title{
Microfadeometry of Face-Mounted and Unmounted Chromogenic Photographs
}

Shannon Brodgon-Grantham ${ }^{1}$, Stephanie Lussier ${ }^{2}$. Christopher A. Maines ${ }^{3}$, Molly McGath ${ }^{3,4}$, and Thomas Lam ${ }^{1}$

${ }^{1}$ Museum Conservation Institute, Smithsonian Institution, Suitland, MD, USA

${ }^{2}$ Hirshhorn Museum and Sculpture Garden, Smithsonian Institution, Washington, DC, USA

${ }^{3}$ National Gallery of Art, Washington, DC, USA

${ }^{4}$ Heritage Conservation Science Laboratory, Johns Hopkins University, Baltimore, MD, USA

Chromogenic photographs possess a multi-laminate structure that consists of three discrete dye layers, cyan, magenta, and yellow, which form a full color image, on top of a polyethylene coated paper support. The application of face-mounting is an optional aesthetic finishing technique in the photograph and graphics industry and involves the irreversible mounting of an optically transparent or translucent sheet of acrylic to the recto of a photograph or print using a clear, construction-grade silicone rubber adhesive. Chromogenic photographs are commercially very common and are frequently found within museum collections; microfadeometry was performed to learn about their light sensitivity.

The chromogenic photographic sample used in this study is shown in Fig. 1. Since all colors on the chromogenic photographs used in this study are made from the mixture of cyan, magenta, and yellow, the focus of this study was to test the light sensitivity of those three colors as well as a non-colored area (white) on the primary support (Fig. 1) with the selected regions of interest shown in black circles. An unmounted and a face-mounted sample were evaluated. The face-mounted sample was mounted with Acrylite FF3 acrylic and General Electric SCS1000 silicone. Microfadeometry is a technique that measures the changes in visible reflectance (400-700nm) of a material in real time using the 1976 CIELAB color space values in order to assess the light-stability of the material. Because of the small size of the test spot (analytical spot is $3 \mathrm{~mm}$ and potential degradation spot is $0.2-0.4 \mathrm{~mm}$ ), and that the ability to stop measurement in real-time well-before it causes visible damage, the technique can be considered a nondestructive test method. Fading measurements were performed with the Oriel 80190 Fading Test System (Newport Oriel Corporation) that is equipped with an IR filtered Xe lamp light source and the visible reflected light from the spot is collected by a fiber optic taken to the spectrometer unit, and all measurements were carried out for 300 seconds. Color change was calculated using $\Delta E^{*}=$ $\sqrt{\left(L_{0}^{*}-L_{t}^{*}\right)^{2}+\left(a_{0}^{*}-a_{t}^{*}\right)^{2}+\left(b_{0}^{*}-b_{t}^{*}\right)^{2}}$, where the original $\mathrm{L}^{*}, \mathrm{a}^{*}, \mathrm{~b}^{*}$ values at time zero are $L_{0}^{*}, a_{0}^{*}, b_{0}^{*}$ and $L_{t}^{*}, a_{t}^{*}, b_{t}^{*}$ are values at a given time elapsed. A $\Delta \mathrm{E}^{*} \approx 2.3$ units has been reported in the literature to be the just noticeable difference (JND), a values less than 2.3 would not be noticeable by the naked eye [1]. The assessment of the relative kinetics of fading was performed by comparison of the material with different grades of blue wool standards (Light Fastness Standards ISO/BS SDL Atlas USA, Rock Hill). The JND for blue wool standards (blue wool 1(BW1) and blue wool 2 (BW2)) have been established and are classified into ASTM categories for lightfastness [2].

Fig. 2. shows the change of color in $\Delta \mathrm{E} /$ microfading for the primary support (non-colored white region) in comparison with the BW1 and BW2 for both unmounted (solid line) and face-mounted (dashed line) samples. Fig. 3 shows the microfading data for cyan, magenta, and yellow in comparison to BW1 and BW2 for both unmounted (solid line) and mounted (dashed line) samples. Face-mounted samples were found to be less light sensitive and a detailed explanation regarding the color observed by the spectrometer for the different cases of unmounted and face-mounted samples will be presented. 


\section{References:}

[1] M. Mahy et al., Color Research \& Application 19 (1994), 105-121.

[2] http://www.drb-mattech.co.uk/uv\%20blue\%20wool.html

[3] This project was part of a Smithsonian Postgraduate Fellowship in Conservation of Museum Collections based at the Hirshhorn Museum and Sculpture Garden.

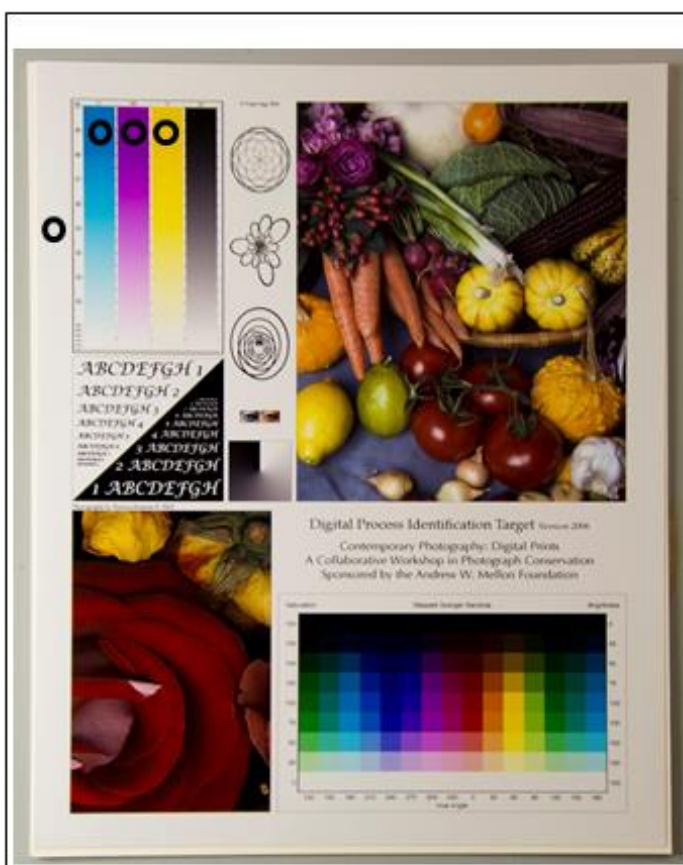

Fig. 1. Sample of an $21.59 \mathrm{~cm}$ by $27.94 \mathrm{~cm}$ chromogenic photograph which is a 2007 version of the American Institute for ConservationPhotographic Materials Group (AIC/PMG) Digital Print Sample Set with the selected regions of interest (in circles) that was microfade tested for unmounted and face-mounted photographs.

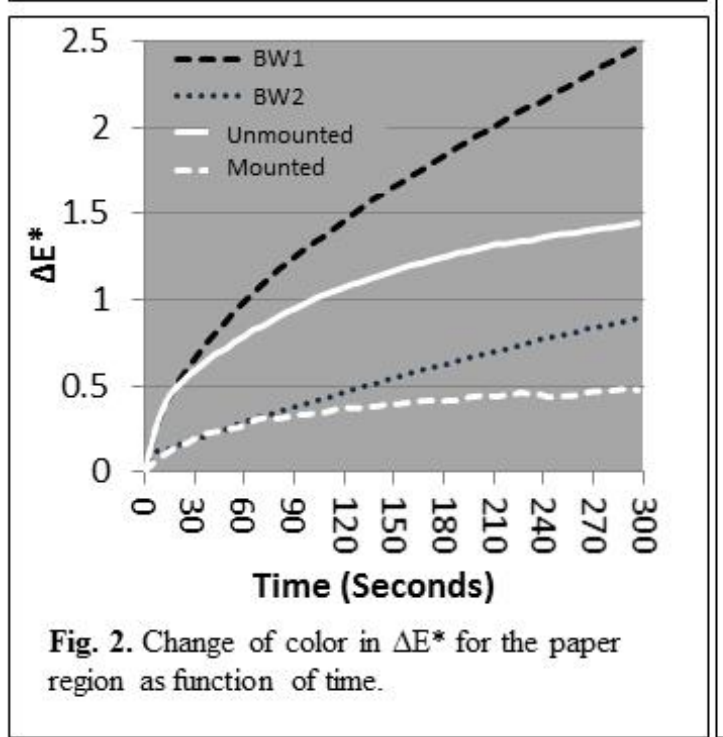

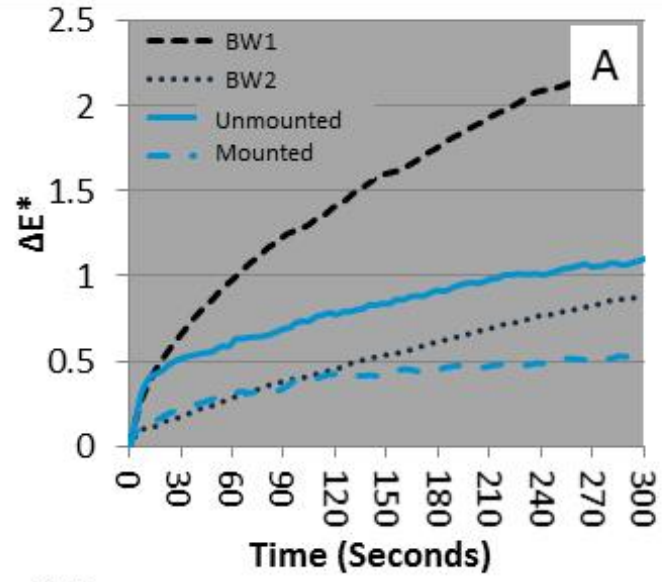

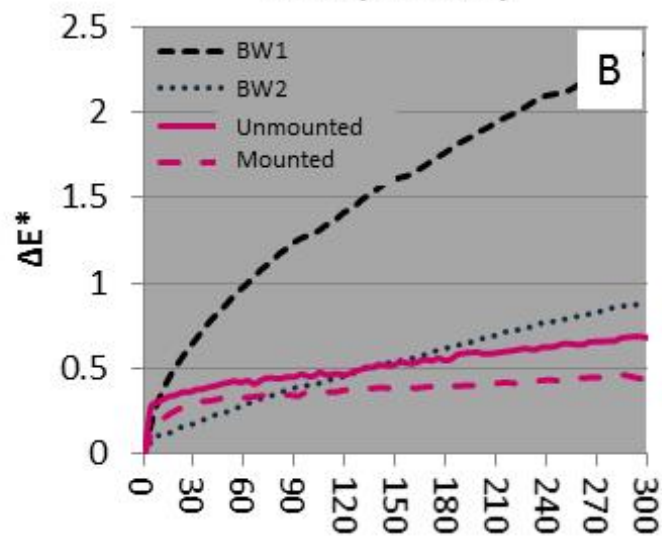

Time (Seconds)

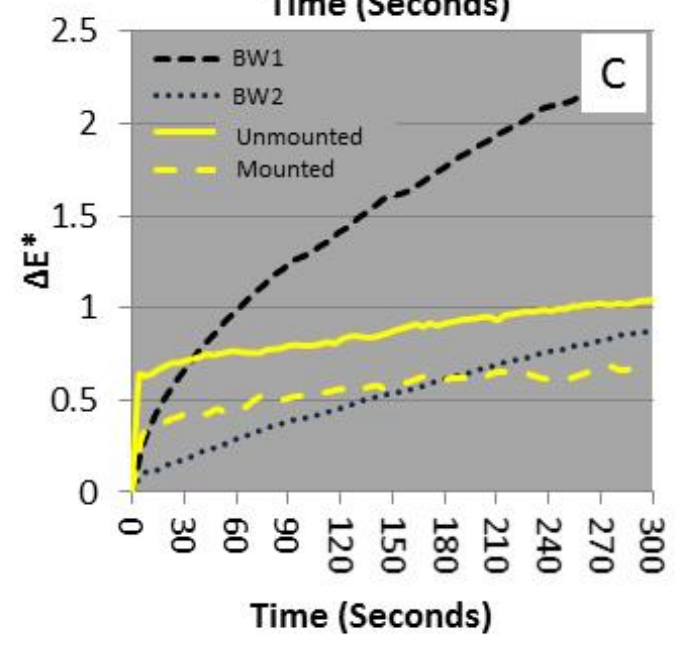

Fig. 3. Change of color in $\Delta E^{*}$ for the (A) cyan, (B) magenta, (C) and yellow color region as function of time. 